Article

\title{
Experimental Study on the Performance of a Novel Compact Electrostatic Coalescer with Helical Electrodes
}

\author{
Yi Shi ${ }^{1,2}$, Jiaqing Chen ${ }^{1, *}$ and Zehao Pan ${ }^{3}$ \\ 1 Beijing Key Laboratory of Pipeline Critical Technology and Equipment for Deepwater Oil \& Gas \\ Development, School of Mechanical Engineering, Beijing Institute of Petrochemical Technology, \\ Beijing 102617, China; shiyi@bipt.edu.cn \\ 2 Beijing Academy of Safety Engineering and Technology, Beijing 102617, China \\ 3 Beijing Zhongyuan Energy. Co., Ltd., Beijing 100101, China; Panzehao2404@163.com \\ * Correspondence: jiaqing@bipt.edu.cn; Tel.: +86-010-812-92136 (ext. 806)
}

Citation: Shi, Y.; Chen, J.; Pan, Z.

Experimental Study on the

Performance of a Novel Compact Electrostatic Coalescer with Helical Electrodes. Energies 2021, 14, 1733. https://doi.org/10.3390/en14061733

Academic Editors: Jalel Azaiez and Edo Boek

Received: 10 December 2020

Accepted: 16 March 2021

Published: 20 March 2021

Publisher's Note: MDPI stays neutral with regard to jurisdictional claims in published maps and institutional affiliations.

Copyright: (c) 2021 by the authors. Licensee MDPI, Basel, Switzerland. This article is an open access article distributed under the terms and conditions of the Creative Commons Attribution (CC BY) license (https:// creativecommons.org/licenses/by/ $4.0 /)$.
Abstract: As most of the light and easy oil fields have been produced or are nearing their end-life, the emulsion stability is enhanced and water cut is increasing in produced fluid which have brought challenges to oil-water separation in onshore and offshore production trains. The conventional solution to these challenges includes a combination of higher chemical dosages, larger vessels and more separation stages, which often demands increased energy consumption, higher operating costs and larger space for the production facility. It is not always feasible to address the issues by conventional means, especially for the separation process on offshore platforms. Electrostatic coalescence is an effective method to achieve demulsification and accelerate the oil-water separation process. In this paper, a novel compact electrostatic coalescer with helical electrodes was developed and its performance on treatment of water-in-oil emulsions was investigated by experiments. Focused beam reflectance measurement (FBRM) was used to make real-time online measurements of water droplet sizes in the emulsion. The average water droplet diameters and number of droplets within a certain size range are set as indicators for evaluating the effect of coalescence. We investigated the effect of electric field strength, frequency, water content and fluid velocity on the performance of coalescence. The experimental results showed that increasing the electric field strength could obviously contribute to the growth of small water droplets and coalescence. The extreme value of electric field strength achieved in the high-frequency electric field was much higher than that in the power-frequency $(50 \mathrm{~Hz})$ electric field, which can better promote the growth of water droplets. The initial average diameters of water droplets increase with higher water content. The rate of increment in the electric field was also increased. Its performance was compared with that of the plate electrodes to further verify the advantages of enhancing electrostatic coalescence and demulsification with helical electrodes. The research results can provide guidance for the optimization and performance improvement of a compact electrocoalescer.

Keywords: electrocoalescence; electrode design; compact separator; oil-water separation; water-inoil emulsion

\section{Introduction}

As the oil well's production life increases, the water content in the oil also grows. It is usually in the form of water-in-oil (W/O) emulsion or oil-in-water $(\mathrm{O} / \mathrm{W})$ emulsion, which depends on the value of water cut. The existence of such emulsions brings challenges in offshore crude oil production. Firstly, the large amount of water and oil phase can easily form the water in oil emulsion and in the colloidal state because of its high stability and viscosity during multiphase transportation, which causes substantial transport problems. Secondly, the process of removing water at the platform is usually achieved in large horizontal separators with the help of gravity and demulsifying chemicals. It is time-consuming and expensive to make the water content below $0.5 \%$ during the treatment 
when the water cut reaches more than $90 \%$. Therefore, it is necessary to use more effective and compact methods to separate water from oil [1]. Nowadays, possible separation methods of water-in-oil emulsions include gravity or centrifugal settling [2,3], thermal treatment, chemical demulsification [4-6], $\mathrm{pH}$ adjustment [7], electrostatic demulsification [8-10], the freeze/thaw method, filtration, membrane separation, microwave radiation and ultrasonic energy, etc. [11-14]. Compared with the other widely used or tried solutions, electrostatic demulsification is becoming more and more popular, both from environmental and economical points of view [12].

Generally, it involves three stages for the water-droplet pair electrocoalescence in $\mathrm{W} / \mathrm{O}$ emulsion. Firstly, the droplets should approach each other and be very close under the action of various long-range flocculation forces. Secondly, the drainage or thinning of the film occurs to reduce the interfacial area. Once the film thickness reaches a critical value, the breakdown or rupture of the film will be easily achieved if there is some instability or disturbance [11]. Urdahl et al. [15] and Harpur et al. [16] investigated electrostatic destabilization of water-in-oil emulsions with a water content up to $30 \%$ under flowing conditions in the laboratory. The experimental results showed that the droplet size increased up to a factor of 10 after flowing through the rectangular electrocoalescer. Since the late 1990s, the electrocoalescence technology has received extensive attention and development, in order to further improve the separation efficiency of the horizontal gravity separator and the electric dehydrator and reduce the overall investment, construction and operating costs of facilities. Various types of electrostatic coalescers have been developed [17-19]. The compact electrostatic coalescer (CEC) [20] and inline electro coalescer (IEC) [21,22] were the typical representatives. From the perspective of their working mechanisms, they both involve electrostatic coalescence and demulsification characteristics of $\mathrm{W} / \mathrm{O}$ emulsions under non-uniform electric fields. The effect of the electric field is mainly related to the properties of the oil-water phase and the type of electrode [23]. Flow conditions could also affect the electrocoalescence process. In the common commercial horizontal electrocoalescer, it usually obtains the laminar flow condition of the fluid and the gravity is set as the primary action [24]. Atten [25] found that the electric coalescence efficiency of water droplets in $\mathrm{W} / \mathrm{O}$ emulsion under moderate turbulence flow condition is better than that in the laminar flow condition. Urdahl et al. [26] also pointed out that turbulence contributes to droplet coalescence. However, the droplet will be dispersed or broken-up if the turbulent intensity reaches a certain value. There is an optimal turbulence intensity that could make full use of the positive effects of disrupting the water-droplet chains whilst avoiding the negative effects of dispersing the larger droplets. Melheim and Matteo [27] studied a model of a turbulent electrocoalescer numerically in the Eulerian-Lagrangian framework. The results showed that the collision frequency is mainly controlled by the turbulence and turbulent electrocoalescence works well for all simulated water contents. Li et al. [28] experimentally studied the demulsification efficiency of $\mathrm{W} / \mathrm{O}$ emulsion by combining with centrifugal and electrical fields in a novel device. The influence of electric frequency, voltage and rotational speed on the separation efficiency were both investigated. Nath [29] investigated the dynamics of viscoplastic droplets under the combined action of electric field and shear flow by performing direct numerical simulations. The simulations reveal that the relative competition of shear, electric, and surface tension forces ultimately decides the mechanism of the droplet disintegration. Ismail et al. [30] developed a compact high-performance electrostatic coalescer and made tests on an Arab medium crude oil under different process conditions, including different temperatures and electric field strengths. Several static mixers with different geometries were housed in the compact inline coalescer chamber and tested for their properties in the electrostatic-enhanced demulsification process. The results show that installing a static mixer in the electrostatic coalescer cell can improve the oil dehydration performance by creating a favorable mild turbulence flow. Sameer and Rochish [31] used different kinds of electrode systems such as quadrupole, pin-plate, four-pin and annular electrodes to generate the non-uniform electric fields. The coalescence behavior of emulsions in the non-uniform electric fields was analyzed and the results 
were compared with those in a uniform field. The results showed that in $\mathrm{W} / \mathrm{O}$ emulsion, an asymmetric non-uniform field generated using the pin-plate electrodes is found to be advantageous as compared to the uniform and symmetric quadrupole non-uniform electric fields. Luo et al. [32] experimentally studied the effect of field nonuniformity on the electrocoalescence of $\mathrm{W} / \mathrm{O}$ emulsion under different electric field configurations including coaxial cylindrical electrodes, pin-plane electrodes and parallel plate electrodes through microscopic observation and image analysis. The results showed that non-uniform fields can enhance the performance of electrocoalescence compared to uniform fields due to the combined effects of dipole-dipole interaction and dielectrophoresis. Li et al. [33] studied the performance of a novel electrode geometry which is formed into a V-shape and generates non-uniform electric field. The performance of this novel electrode was investigated for different electrical parameters, fluid physical properties and residence time by experiment and numerical simulation. The results showed that the electrocoalescence of $\mathrm{W} / \mathrm{O}$ emulsion could be enhanced since the electrical attractive force between neighboring droplets is promoted with the compact design. Zhou et al. [34] investigated the effect of dielectrophoresis on the coalescence of binary droplets under a non-uniform electric field. The results showed that the effects of dielectrophoresis on coalescence vary with different electrodes. For positive dielectrophoresis, there is a critical distance of coalescence and it is affected by the electric field intensity, continuous phase viscosity, and droplet diameter. Gong et al. [35] studied the separation performance of a demulsification and dewatering device coupling electric and centrifugal fields by numerical and experimental methods. The results indicate that numerical results obtained by computational fluid dynamic coupling with a population balance model (PBM) are in good accordance with experiments. Furthermore, the effects of droplet dynamic characteristics cannot be ignored in the oil-water separation process. Due to the structure limitation of the annular flow channel in CEC and rectangular flow channel in IEC, it is difficult to sufficiently promote the collision and coalescence of the dispersed water droplets in crude oil emulsions under suitable flow conditions. Therefore, there is still great potential to enhance the demulsification and coalescence efficiency.

This paper is based on the fact that proper turbulence can promote electrostatic coalescence. The electrode structure is changed to generate a suitable flow condition and to sufficiently promote the electrostatic coalescence of water droplets by fully take advantages of the flow conditions and electric fields. A novel compact electrostatic coalescer with helical electrodes is developed. The residence time could be prolonged and the turbulence flow condition is produced in the helical channel. Thus, the electrocoalescence is promoted. The performance of novel compact electrostatic coalescer is experimentally studied and the influence of the electric field strength, frequency, W/O emulsion velocity and water content in the emulsion on the demulsification are investigated. The experimental results are compared with those of the electrostatic coalescer with parallel plate electrodes.

\section{Geometry of the Novel Electrostatic Coalescer}

The structure of the novel compact electrostatic coalescer is shown in Figure 1. It mainly contains the helical electrodes, insulated central shaft, insulated cylinder, fluid inlet and outlet and the part to connect a high voltage line. The helical electrodes are composed by helical blades and mounted alternately. They are separately connected to the high-power voltage and the ground. A nonuniformed electric field is generated between the helical electrodes. Meanwhile, the W/O emulsions flow through the helical channel between the two types of electrodes. It puts the emulsion into a transitional flow or weakly turbulent flow conditions, which contributes to increasing the collision rate of water droplets and thus promotes the demulsification coalescence efficiency. 


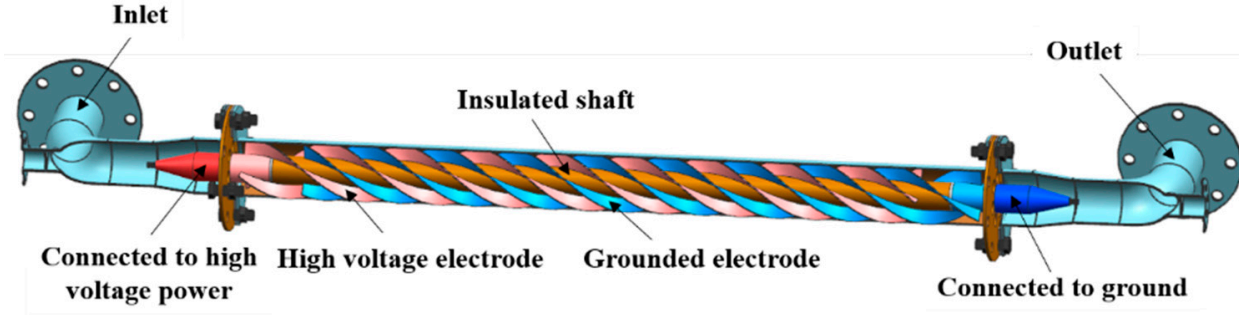

(a)

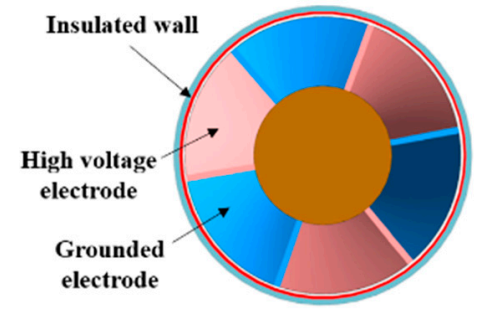

(b)

Figure 1. Structure of compact electrostatic coalescer with helical electrodes. (a) 3D assembly model; (b) cross-section view of the assembly.

The design flowrate of the compact electrostatic coalescer is $2 \mathrm{~m}^{3} / \mathrm{h}$. The inlet and outlet pipe diameters are both $12.7 \mathrm{~mm}$. The main geometry parameters are shown in Table 1. There are four helical blades and the pitch between each blade is $35 \mathrm{~mm}$. Figure 2 shows a picture of the compact electrostatic coalescer. The demulsification performance in the uniformed electric field generated by parallel plate electrodes will be compared with that of the helical electrodes.

Table 1. The main parameters of compact electrostatic coalescer.

\begin{tabular}{cc}
\hline Parameters & Value \\
\hline Design volume flowrate $\left(\mathrm{m}^{3} / \mathrm{h}\right)$ & 2 \\
Inlet diameter $(\mathrm{mm})$ & 12.7 \\
Outlet diameter $(\mathrm{mm})$ & 12.7 \\
Outlet diameter of helical blade $(\mathrm{mm})$ & 30 \\
Inner diameter of helical blade $(\mathrm{mm})$ & 18 \\
Thickness of helical blade $(\mathrm{mm})$ & 3 \\
Length of the helical channel $(\mathrm{mm})$ & 500 \\
\hline
\end{tabular}

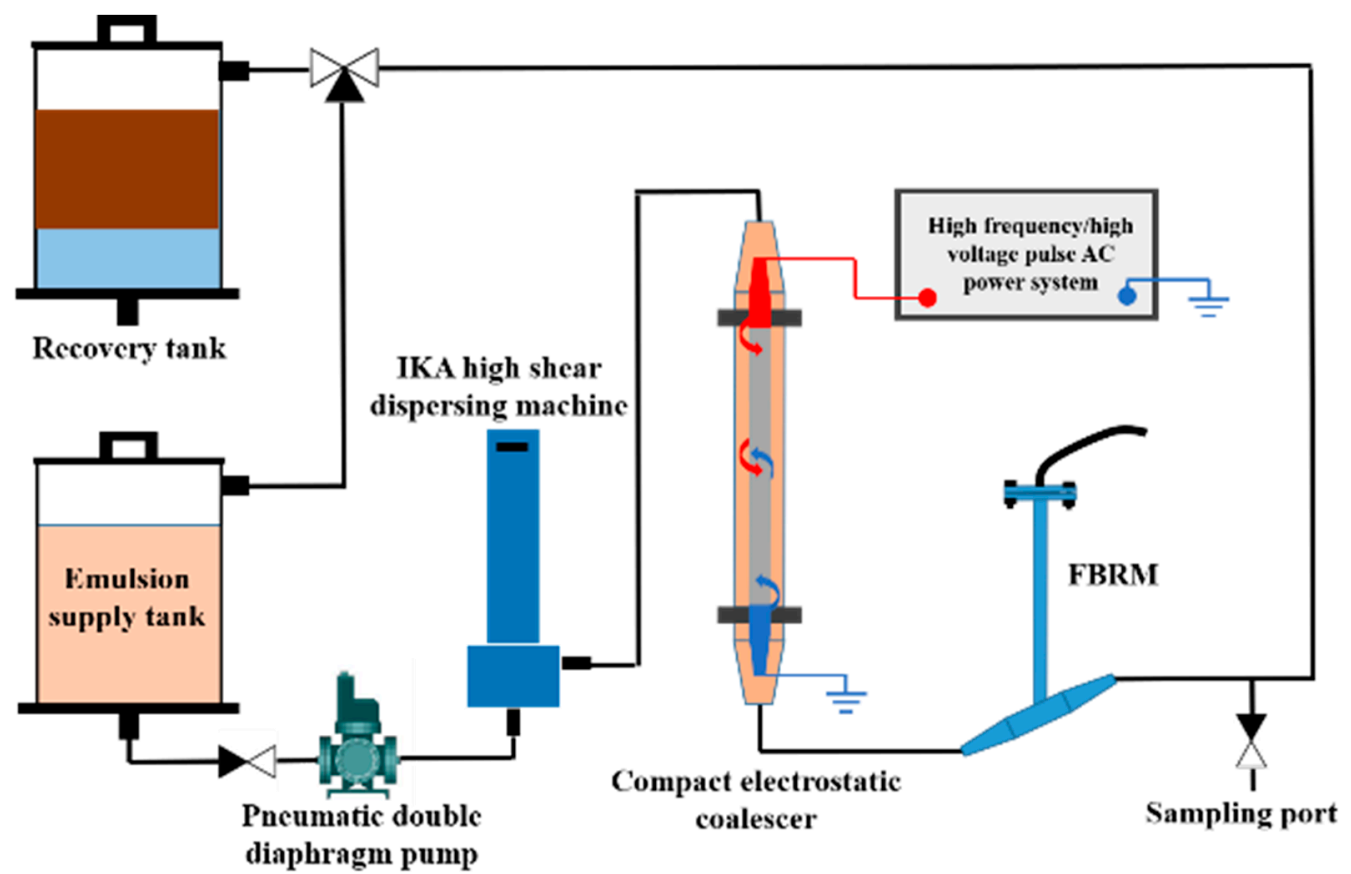

Figure 2. Experimental chart of test system. 


\section{Experimental Procedure}

The experimental chart of the compact electrostatic coalescer is shown in Figure 2. It contains the emulsion supply tank, pneumatic double diaphragm pump, IKA high shear dispersing machine, FBRM, waste liquid recovery tank, etc. The compact electrostatic coalescer is installed between the IKA high shear dispersing machine and FBRM. The diameters and numbers of water droplets in W/O emulsion could be tracked in real-time by the FBRM. The range of droplet diameters that FBRM can measure is $0.5 \mu \mathrm{m}$ to $3 \mathrm{~mm}$. Figure 3 is a picture of the experiment system. Figure 4 shows the high frequency/high voltage pulse AC power system. It contains the GFG-3015 function generator, Trek Model 10/40A-HS high voltage amplifier and S-620 $20 \mathrm{MHz}$ oscilloscope. The function generator can provide a voltage of 0.01 to $10 \mathrm{~V}$ and a $10 \mathrm{kHz}$ to $15 \mathrm{MHz}$ frequency. It can also produce different AC or DC waveforms including rectangular wave, sine wave, triangle wave and sawtooth wave. The voltage amplifier can amplify the voltage signal to as high as 1000 times and output 0 to $10 \mathrm{kV}$ voltage whilst keeping the frequency and waveforms constant.

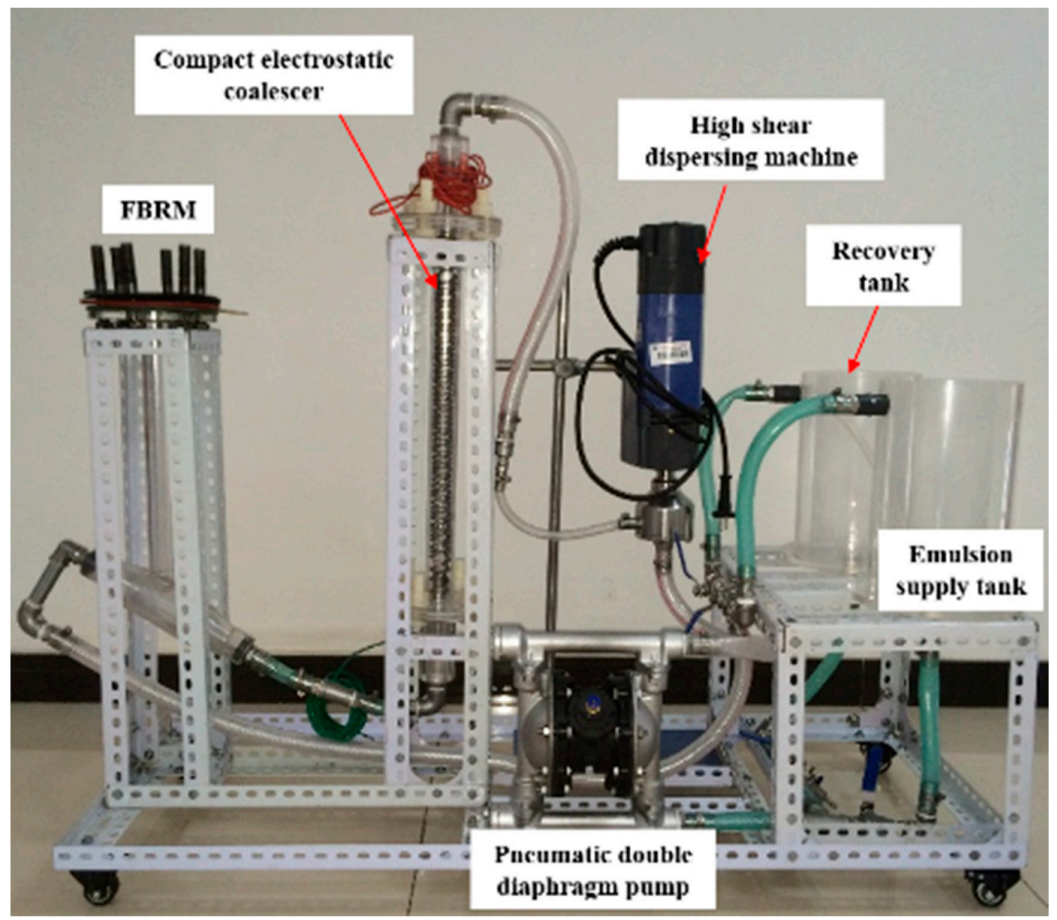

Figure 3. Picture of the experiment system.

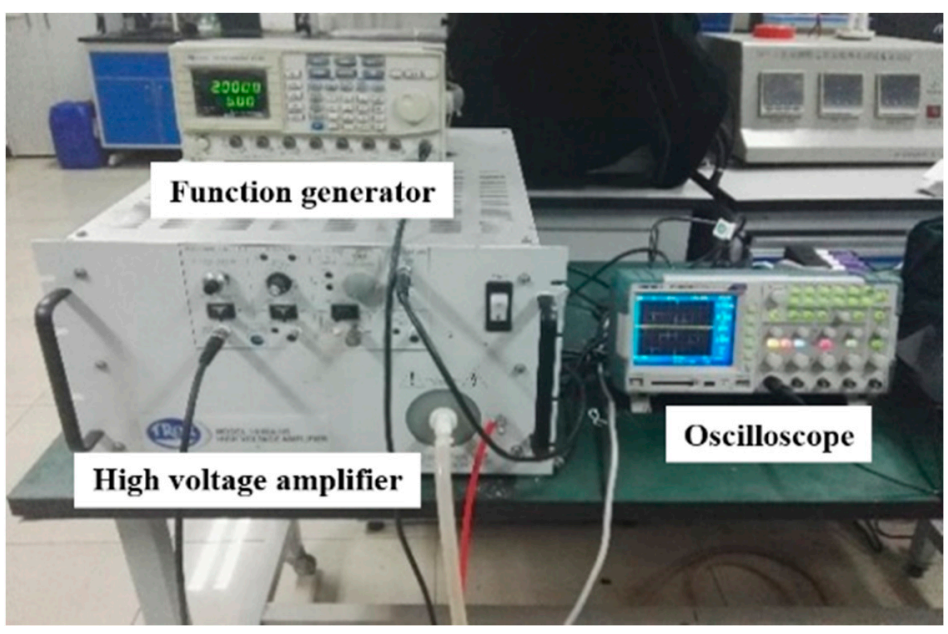

Figure 4. High frequency/high voltage pulse AC power system. 
During the experiment, the $\mathrm{W} / \mathrm{O}$ simulated emulsion was generated by using 10\# white oil, water and Span 80 emulsifier. Firstly, a certain amount of 10\# white oil was fed into the emulsion supply tank and the oil was allowed to circulate with a constant flowrate in the experimental process by starting the pneumatic double diaphragm pump. Then, the IKA high shear dispersing machine was started with a certain rotational speed and water and emulsifier was injected into the emulsion supply tank in proportion to generate the $\mathrm{W} / \mathrm{O}$ emulsion. When the generated emulsion stabilized, the high frequency/high voltage pulse AC power was turned on and specific electric field parameters were set. The variations of the distribution of the water droplet diameters were collected by the FBRM in real-time and were used to evaluate the performance of the compact electrostatic coalescer. FBRM is the industry-standard measurement technique used for the in-process measurement of particles. A highly precise chord length distribution (CLD), sensitive to particle size and count, was reported in real time without the need for sampling or sample preparation. No shape is assumed and the measurement can be applied at full process concentrations in opaque or translucent slurries and emulsions. The method of online in-situ measurement can reduce the negative impact on the accuracy of the measurement results during the dilution process for the sampling if using the traditional method.

During the experiment, the influence of electric parameters and flow conditions such as electric field strength, frequency, water content and flow velocity on the novel compact electrostatic coalescer performance were investigated. Meanwhile, the performance of the electrostatic coalescer with parallel-plate electrodes was also studied and the results were compared with that of the novel compact electrostatic coalescer.

\section{Results and Discussion}

\subsection{Research on Characteristics of Test Emulsion System}

Since the experiment is conducted by circulating the W/O emulsion in the flow loop with various electric parameters, it is necessary to ensure the reliability and repeatability of experimental results. In order to determine the effect of increased shear time and enhanced emulsion stability on the experimental results of electrostatic coalescence equipment, the experiment was repeated several times with the same $\mathrm{W} / \mathrm{O}$ emulsion and same electric parameters. Figure 5 shows the variations of water droplet numbers in $\mathrm{W} / \mathrm{O}$ emulsion. The vertical axis in Figure 5 represents the number of water droplets within $10 \mu \mathrm{m}$. The horizontal axis in Figure 5 represents the experiment time. The experiment was conducted continuously for $30 \mathrm{~min}$. During the experiment, the electric field was powered on and off several times. The electric field strength is $1.8 \mathrm{kV} / \mathrm{cm}$ and the frequency is $50 \mathrm{~Hz}$. Figure 6 shows the variations of the average droplet diameter. It can be concluded that once the high voltage power is on, water droplets with diameters less than $10 \mu \mathrm{m}$ are rapidly coalesced under the electrical field. Additionally, the number of small water droplets decreased, but decreased slightly as time went on, which is mainly related to the characteristics of the flow-loop system itself. The water and oil emulsions demulsified by the electrostatic coalescer were finally returned to the emulsion supply tank and were mixed with the original emulsion. Thus, the stability of the initial emulsion changed to some extent. When the high voltage power is off, the shearing effect becomes the domain factor again and the emulsion system gradually recovers to its original state. Figure 5 shows that the number of small water droplets rapidly decreases when the electric is powered on at about $200 \mathrm{~s}$. Then, the decrease trend becomes less pronounced. The main reason is that the coalescence effect under the electric field is stronger than the emulsification effect under the high shear dispersing machine. As the electric field action time extends, the actual number of water droplets flowing through the electrostatic coalescer is less than the number when the power is just turned on.

From Figures 5 and 6, it can be concluded that the experiment could be conducted with highly repeatable results within $15 \mathrm{~min}$. Once the experiment time is more than $15 \mathrm{~min}$, the average droplet diameter in the emulsion system and number of small water droplets within $10 \mu \mathrm{m}$ both increases. With the same electric field parameters, the increment of 
the average water droplet diameter and the decrement of the droplet number are kept relatively constant. Based on the above discussion, red dotted lines are used to represent the measured values before and after the power is working. The difference before and after power-on is applied to characterize the demulsification performance under different electric field parameters.

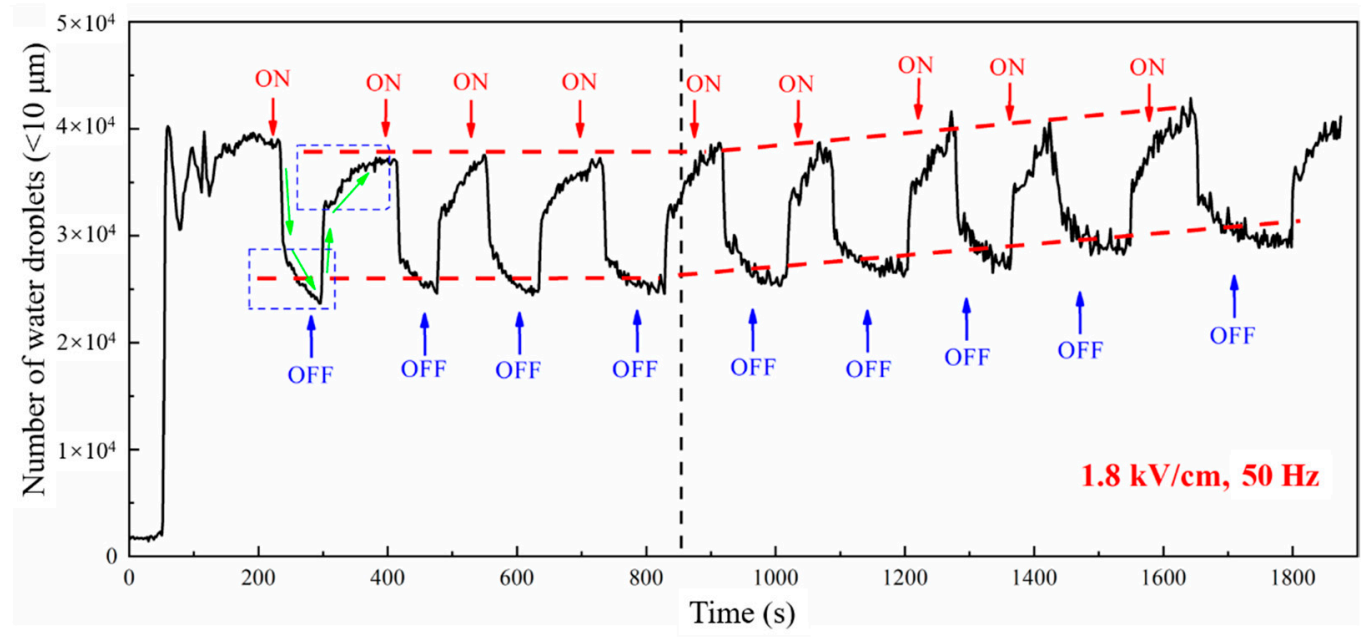

Figure 5. Variations of water droplets number within $10 \mu \mathrm{m}$.

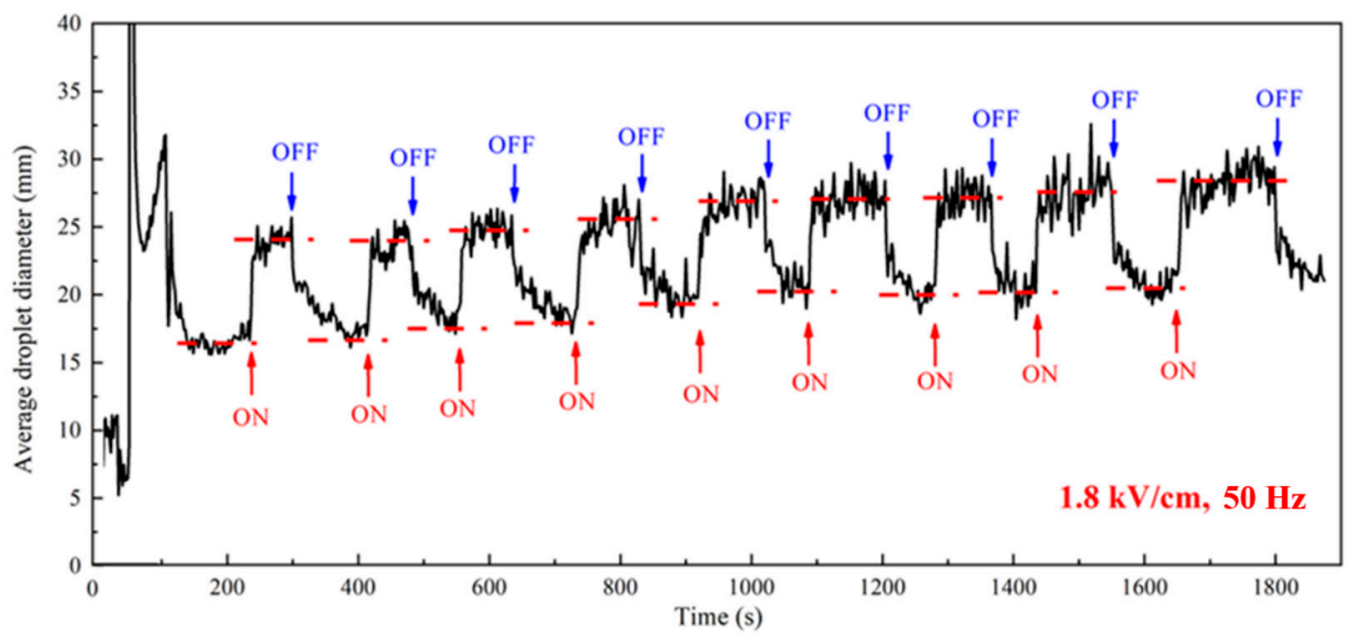

Figure 6. Average water droplet diameters.

\subsection{Effect of the Electric Field Strength}

The flowrate, water content, rotational speed of high shear dispersing machine and other condition parameters were kept constant. The electric frequency was $50 \mathrm{~Hz}$. The coalescence performance with different electric field strengths has been investigated. Parameters are shown in Table 2.

Figure 7 shows the variations of the number of water droplets within $10 \mu \mathrm{m}$ in the emulsion as time increases under different electric field strengths. It can be seen that the device has a short circuit which causes the power supply to start overload protection when the electric field strength is $2 \mathrm{kV} / \mathrm{cm}$. There is a minimum electric field strength which keeps the water droplets within $10 \mu \mathrm{m}$ coalesce. It could not promote the coalescence of small water droplets when the electric field strength was less than $0.8 \mathrm{kV} / \mathrm{cm}$. Once the electric field strength increased to $1.0 \mathrm{kV} / \mathrm{cm}$, the number of small water droplets decreased by $12 \%$ compared to that in the initial conditions. When it reached $1.8 \mathrm{kV} / \mathrm{cm}$, the number of small water droplets decreased by $31 \%$, which shows that increasing the electric field 
strength has a significant effect on promoting the coalescence and growth of small water droplets. Figures 8-11 show the change trend of the number of water droplets with the diameter ranges 10 to $50 \mu \mathrm{m}, 50$ to $100 \mu \mathrm{m}, 100$ to $150 \mu \mathrm{m}$ and 150 to $300 \mu \mathrm{m}$, respectively. It can be concluded that water droplets within $50 \mu \mathrm{m}$ could rapidly coalesce while the number of those range 50 to $300 \mu \mathrm{m}$ clearly increases. It indicates that the demulsification effect is remarkable. It is worth mentioning that the number of water droplets in the range of 50 to $100 \mu \mathrm{m}$ did not rise quickly when the power was off, as shown in Figure 11. It just slowly recovered to the initial state. This demonstrates that the stability of the emulsion system with water particles in the range of 50 to $100 \mu \mathrm{m}$ is the strongest.

Table 2. Parameters to study the influence of electric field strength on demulsification.

\begin{tabular}{|c|c|c|c|c|c|}
\hline \multicolumn{4}{|c|}{ Flow Condition Parameters } & \multicolumn{2}{|c|}{ Electrical Field Parameters } \\
\hline $\begin{array}{l}\text { Velocity } \\
(\mathrm{m} / \mathrm{s})\end{array}$ & $\begin{array}{c}\text { Water Content } \\
(\%)\end{array}$ & $\begin{array}{l}\text { Amount of Emulsifier } \\
(\mathrm{g} / \mathrm{L})\end{array}$ & $\begin{array}{l}\text { Rotational Speed of Shear } \\
\text { Dispersing Machine } \\
\text { (r/min) }\end{array}$ & $\begin{array}{l}\text { Electric Frequency } \\
(\mathrm{Hz})\end{array}$ & $\begin{array}{l}\text { Electric Field Strength } \\
(\mathrm{kV} / \mathrm{cm})\end{array}$ \\
\hline 0.44 & 15 & 7 & 8000 & 50 & $\begin{array}{c}0.4 \\
0.6 \\
0.8 \\
1 \\
1.2 \\
1.4 \\
1.6 \\
1.8 \\
2\end{array}$ \\
\hline
\end{tabular}

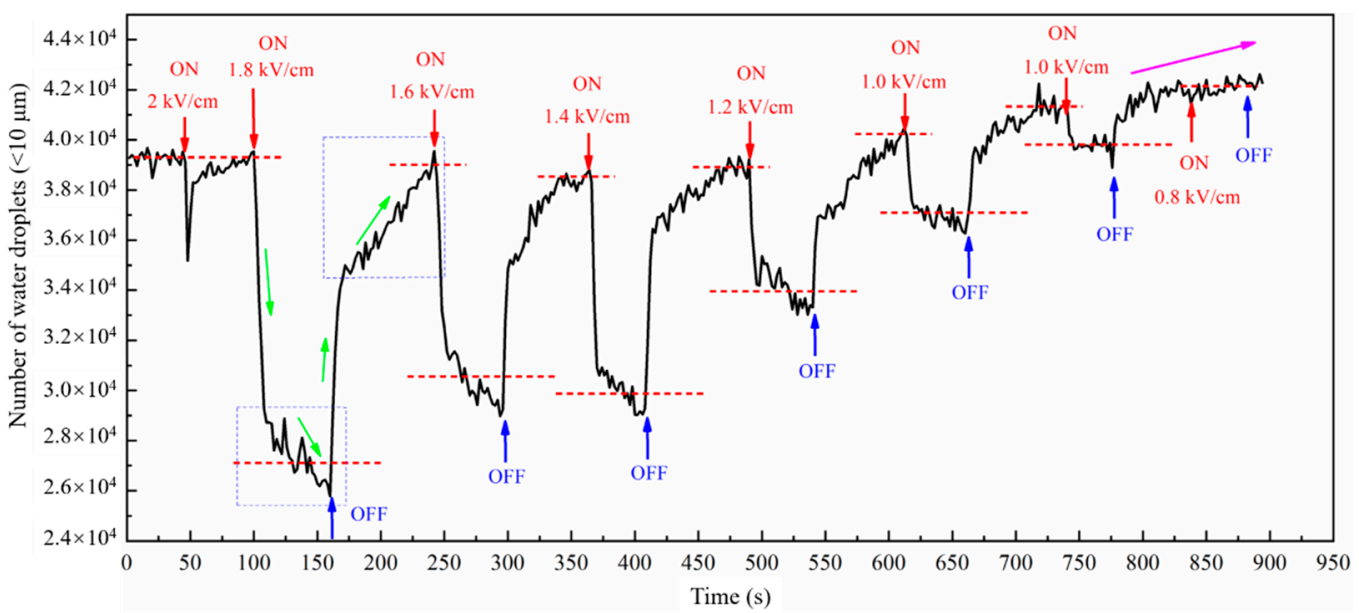

Figure 7. Variations of water droplet number within $10 \mu \mathrm{m}$.

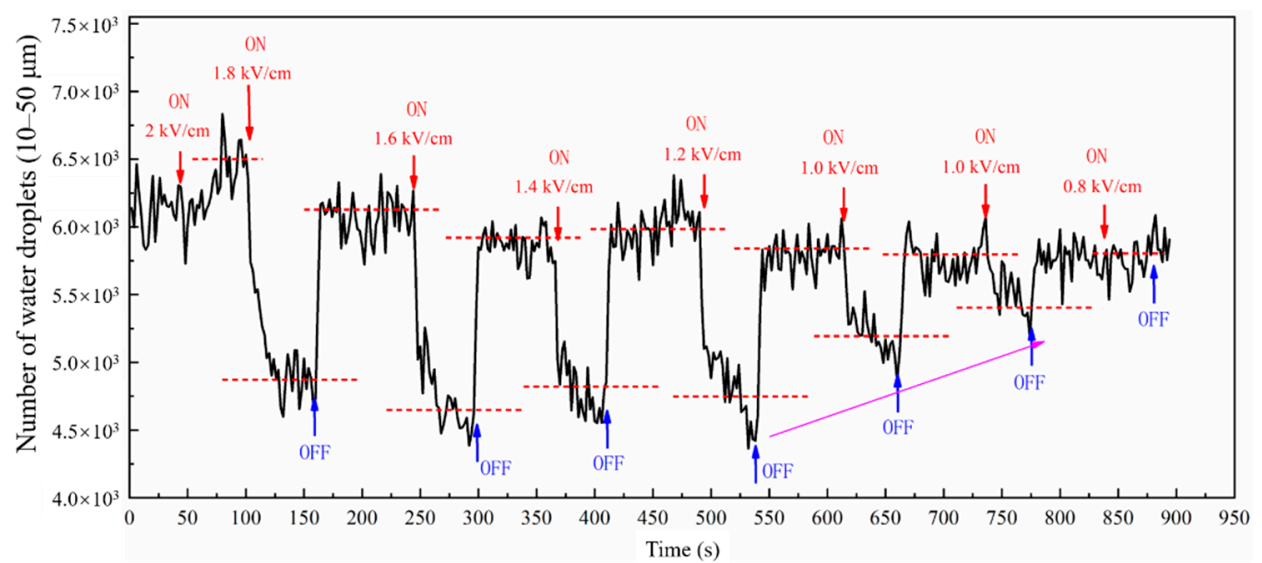

Figure 8. Variations of water droplet number within 10 to $50 \mu \mathrm{m}$. 


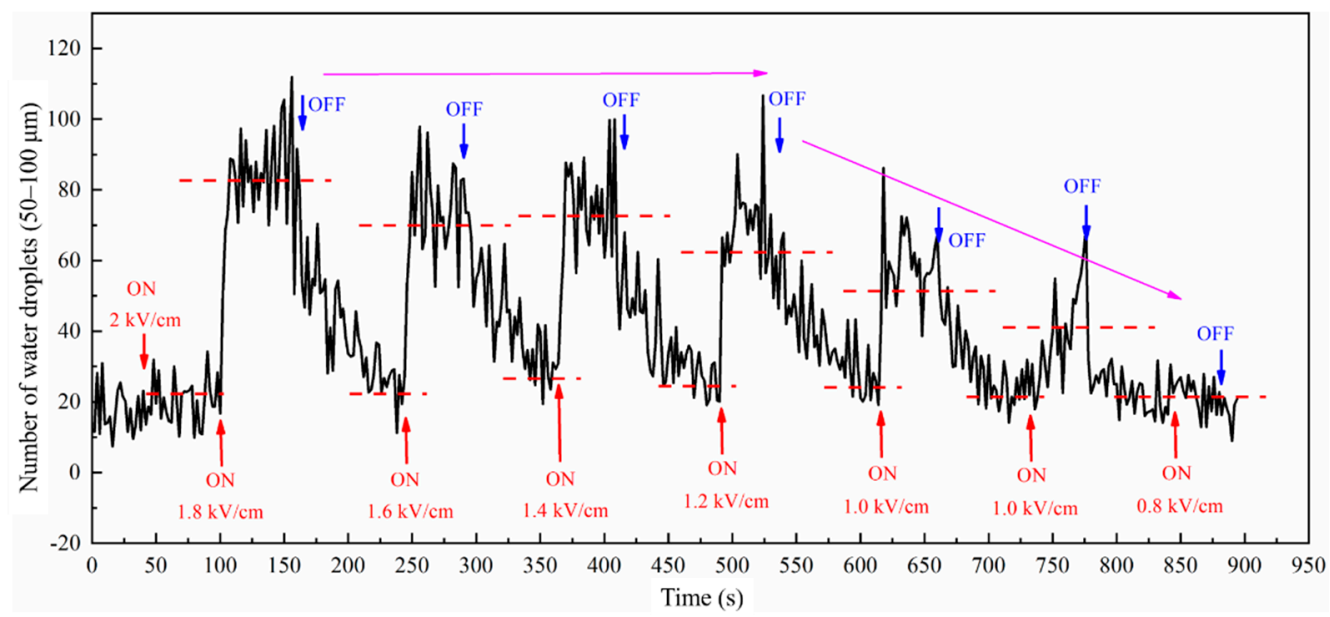

Figure 9. Variations of water droplet number within 50 to $100 \mu \mathrm{m}$.

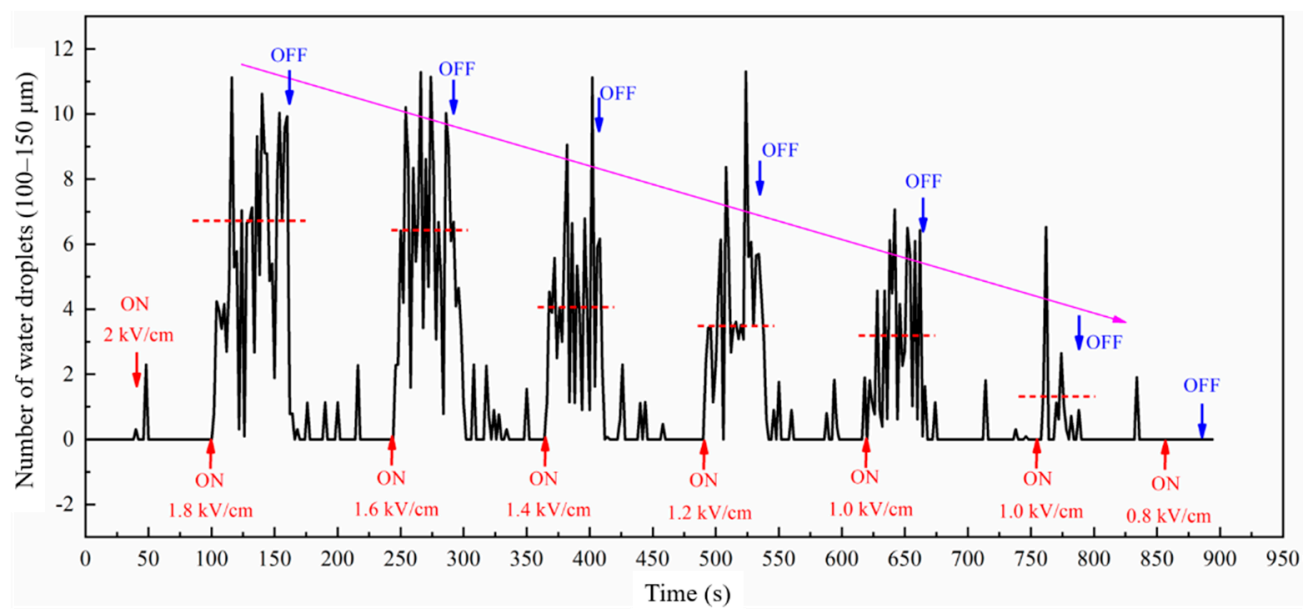

Figure 10. Variations of water droplets number within 100 to $150 \mu \mathrm{m}$.

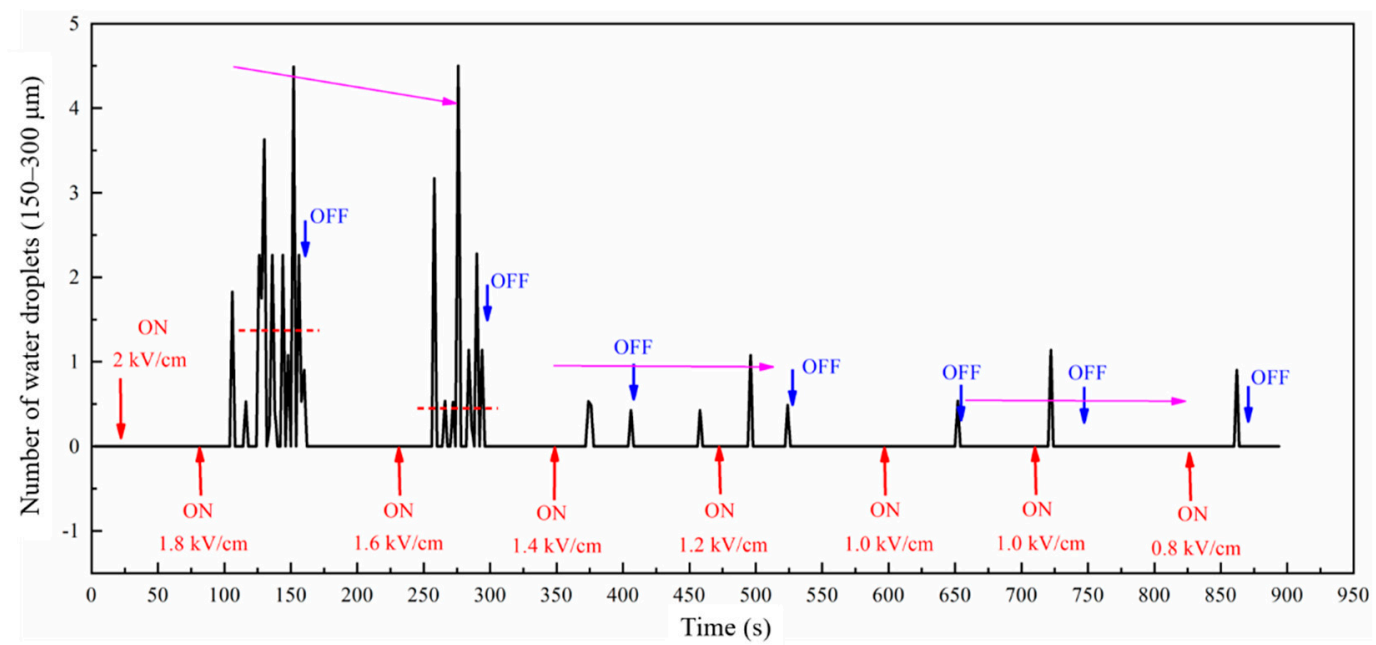

Figure 11. Variations of water droplet number within 150 to $300 \mu \mathrm{m}$.

Figure 12 shows the variations of the average water droplet diameter under different electric field strengths. The initial average water droplet diameter is about $18 \mu \mathrm{m}$. It is rapidly increased in a short of time once the power is on. 


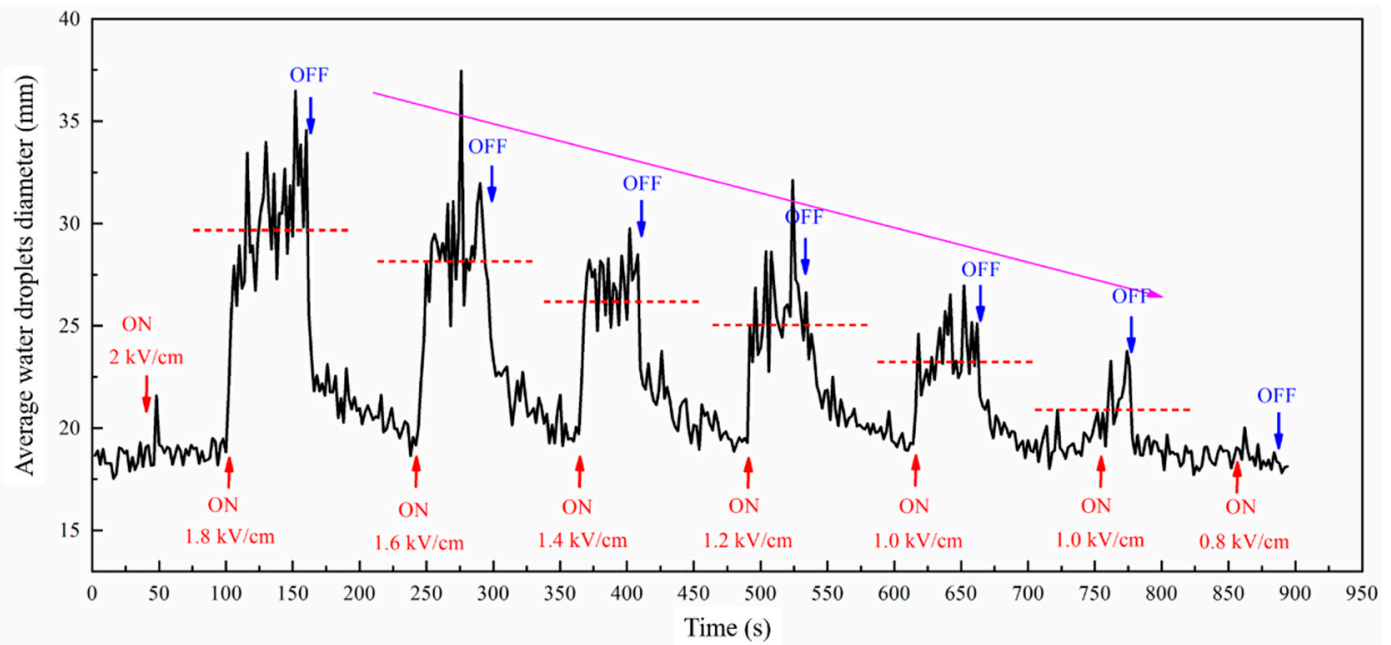

Figure 12. Average water droplet diameters.

\subsection{Effect of the Electric Frequency}

Based on the above studies, the electric field strength is set to $1.8 \mathrm{kV} / \mathrm{cm}$. The effect of the electric frequency on the performance of the compact electrostatic coalescer is investigated in this part. The influence of the electric field strength with high electric frequency $(3000 \mathrm{~Hz})$ has also been studied. The parameters are listed on Table 3.

Table 3. Parameters to study the influence of electric frequency.

\begin{tabular}{|c|c|c|c|c|c|}
\hline \multicolumn{4}{|c|}{ Flow Condition Parameters } & \multicolumn{2}{|c|}{ Electrical Field Parameters } \\
\hline $\begin{array}{l}\text { Velocity } \\
(\mathrm{m} / \mathrm{s})\end{array}$ & $\begin{array}{c}\text { Water Content } \\
(\%)\end{array}$ & $\begin{array}{c}\text { Amount of Emulsifier } \\
(\mathrm{g} / \mathrm{L})\end{array}$ & $\begin{array}{l}\text { Rotational Speed of Shear } \\
\text { Dispersing Machine } \\
(\mathrm{r} / \mathrm{min})\end{array}$ & $\begin{array}{l}\text { Electric Field Strength } \\
(\mathrm{kV} / \mathrm{cm})\end{array}$ & $\underset{(\mathrm{Hz})}{\text { Electric Frequency }}$ \\
\hline & & & & 1.8 & $\begin{array}{c}50 \\
500 \\
1000 \\
1500 \\
2000 \\
2500 \\
3000\end{array}$ \\
\hline 0.44 & 15 & 7 & 8000 & $\begin{array}{l}2.2 \\
2.4 \\
2.6 \\
2.8 \\
3.0 \\
3.1 \\
3.2 \\
4.0 \\
4.8\end{array}$ & 3000 \\
\hline
\end{tabular}

Figure 13 shows the variation of the average water droplet diameter in the emulsion system as time increases. On the left part of this figure, the electric frequency ranges from $50 \mathrm{~Hz}$ to $3000 \mathrm{~Hz}$ while the electric field strength is kept at $1.8 \mathrm{kV} / \mathrm{cm}$. It indicated that the average droplet diameter increases by about $28 \%$ compared to the initial value. The effect of the electric field on the coalescence performance is weakened as the frequency increases. The maximum electric field strength that can be achieved is $1.8 \mathrm{kV} / \mathrm{cm}$ with a frequency of $50 \mathrm{~Hz}$, as shown in Figure 12. It is hard to determine the optimized electric field parameters according to the variation of droplet diameters under that frequency $(50 \mathrm{~Hz})$.

On the right part of Figure 13, the coalescence performance in the compact electrostatic coalescer with a helical electrode under different electric field strengths and high electric frequency $3000 \mathrm{~Hz}$ is investigated to find the optimized value. The results showed that the maximum electric field strength can be as high as $4.8 \mathrm{kV} / \mathrm{cm}$ with a high electric frequency, $3000 \mathrm{~Hz}$, which is much higher than that with $50 \mathrm{~Hz}$. The average droplet diameter increases by about $54 \%$ with an electric field strength of $3 \mathrm{kV} / \mathrm{cm}$. The increase of 
the average water droplet size decreases slightly when further increasing the electric field intensity. However, the electrostatic coalescence effect is not significantly weakened, even if the electric field strength is excessive. It also verifies the superiority of the high frequency electric field in avoiding a short circuit.

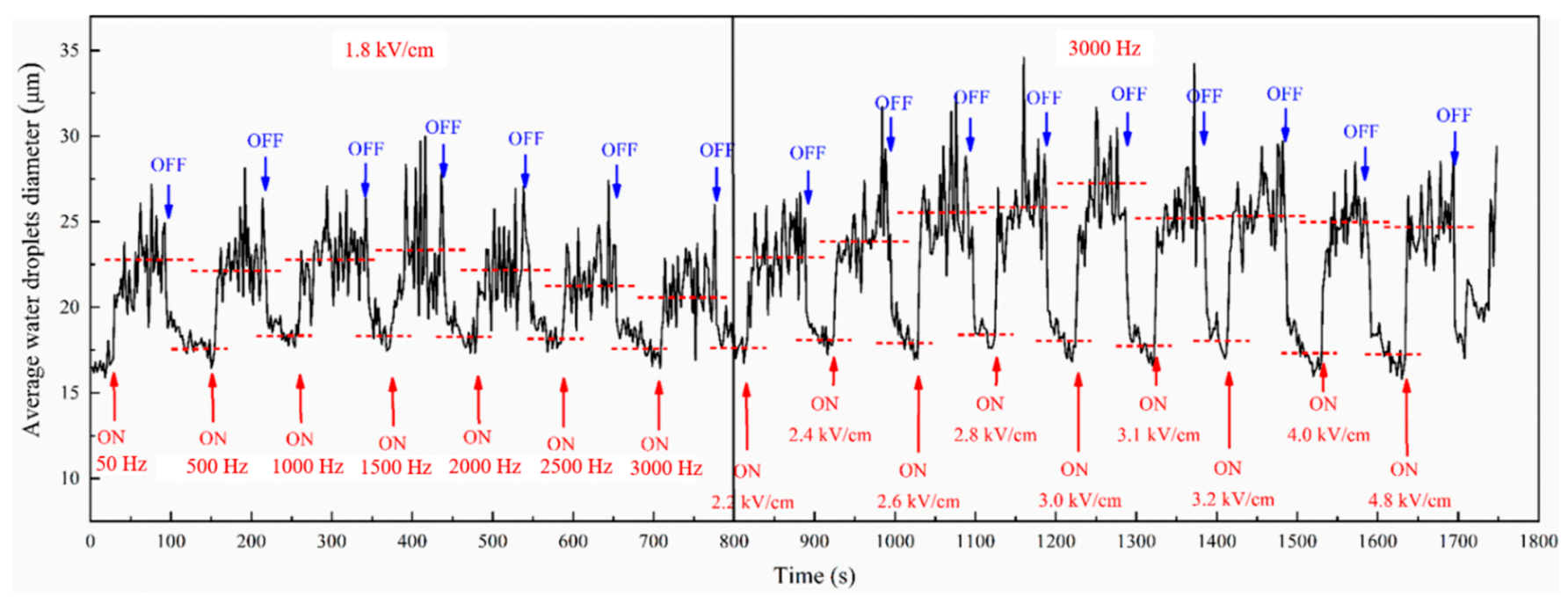

Figure 13. Variations of average water droplet size under different electric field parameters.

Based on the above analysis, the influence of electric field frequency on power consumption is investigated with an electric field strength of $1.8 \mathrm{kV} / \mathrm{cm}$. In the experiment, the power value of the power supply was calculated by recording the actual output average voltage and average current. Figure 14 shows the variation of the average power of the power supply at different frequencies. The average power value is significantly different under various electric field frequencies. As the electric field frequency increases, the average current value decreases and the actual power consumption of the power supply is also reduced. The power consumption at $3000 \mathrm{~Hz}$ is decreased by about $40 \%$ compared to that at $50 \mathrm{~Hz}$. It is lower than the power consumption at $50 \mathrm{~Hz}$, which shows a significant energy-saving effect.

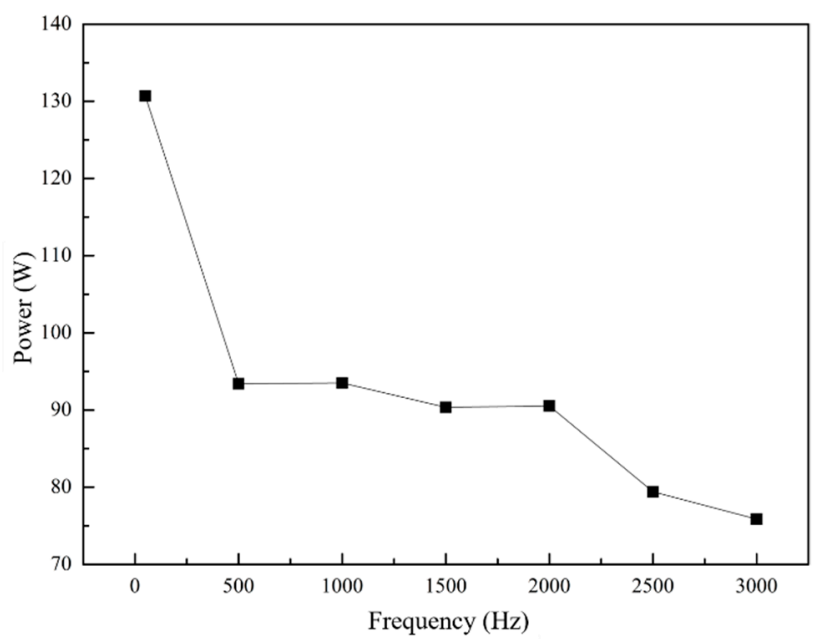

Figure 14. Relationship between power and electric field frequency.

\subsection{Effect of the Water Content}

The emulsification performance of the compact electrostatic coalescer under different emulsion water contents $(10,20,30$, and $40 \%)$ is studied in this part. The amount of 
emulsifier (Span 80) added in the emulsion is $7 \mathrm{~g} / \mathrm{L}$ to better formulate the emulsion. The rotational speed of the high-shear dispersing machine is $8000 \mathrm{r} / \mathrm{min}$. The emulsion flow velocity in the electrostatic coalescer is $0.44 \mathrm{~m} / \mathrm{s}$.

(1) Water content: $10 \%$

Figure 15 shows the variations of average water droplets size in the emulsion with a water content of $10 \%$. Figure 16 shows the number of water droplets within $10 \mu \mathrm{m}$. Comparing the experimental results with a water content of $10 \%$ and those with water a content of $15 \%$ in Figure 13, it can be found that a higher electric field intensity can be achieved at a lower water content. The number of water droplets is greatly reduced and the average droplet size is significantly increased as the electric field intensity increases. However, for emulsions with lower water content, a higher electric field intensity is required to achieve the same average droplet size with high water content.

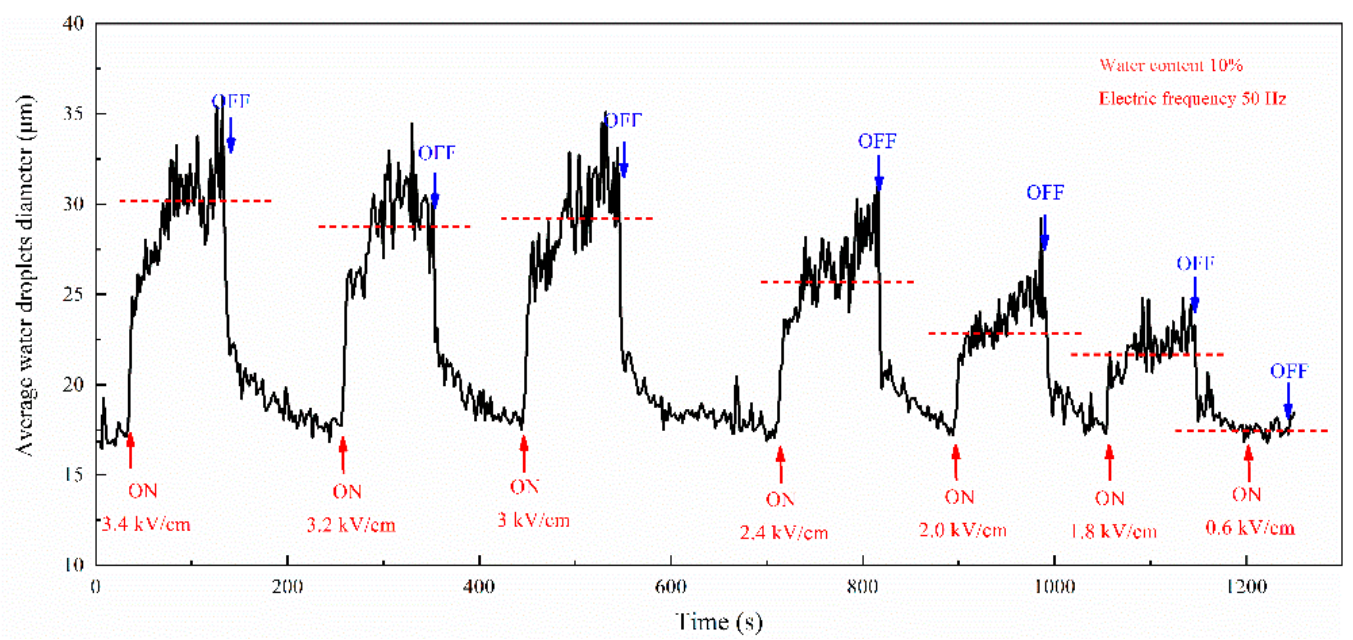

Figure 15. Variations of average water droplet size under different electric field parameters (water content $10 \%$ ).

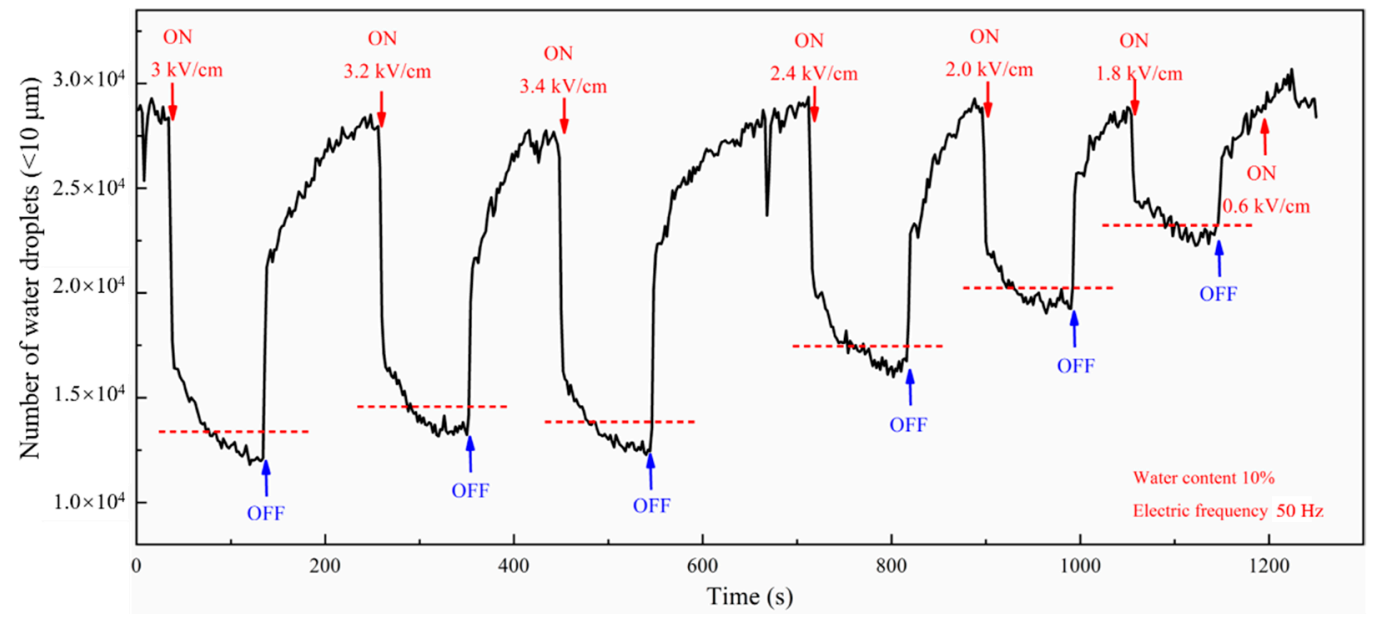

Figure 16. Variations of water droplets number within $10 \mu \mathrm{m}$ (water content $10 \%$ ).

(2) Water content: $20 \%$

Figure 17 shows the variations of the average water droplet size in the emulsion with a water content of $20 \%$ and Figure 18 shows the number of water droplets within $10 \mu \mathrm{m}$. Under the conditions with an electric frequency of $50 \mathrm{~Hz}$, the short circuit occurs as the electric field intensity increases to $1.2 \mathrm{kV} / \mathrm{cm}$. The maximum electric field intensity that can be achieved under a high-frequency electric field $(3000 \mathrm{~Hz})$ is $4.0 \mathrm{kV} / \mathrm{cm}$ and a short 
circuit did not occur under an excessive power voltage. Compared to the demulsification result of the emulsion with a water content of $10 \%$, the initial average droplet size is larger at a water content of $20 \%$ and the increment amplitude of the average droplet size after the coalescence also grows.

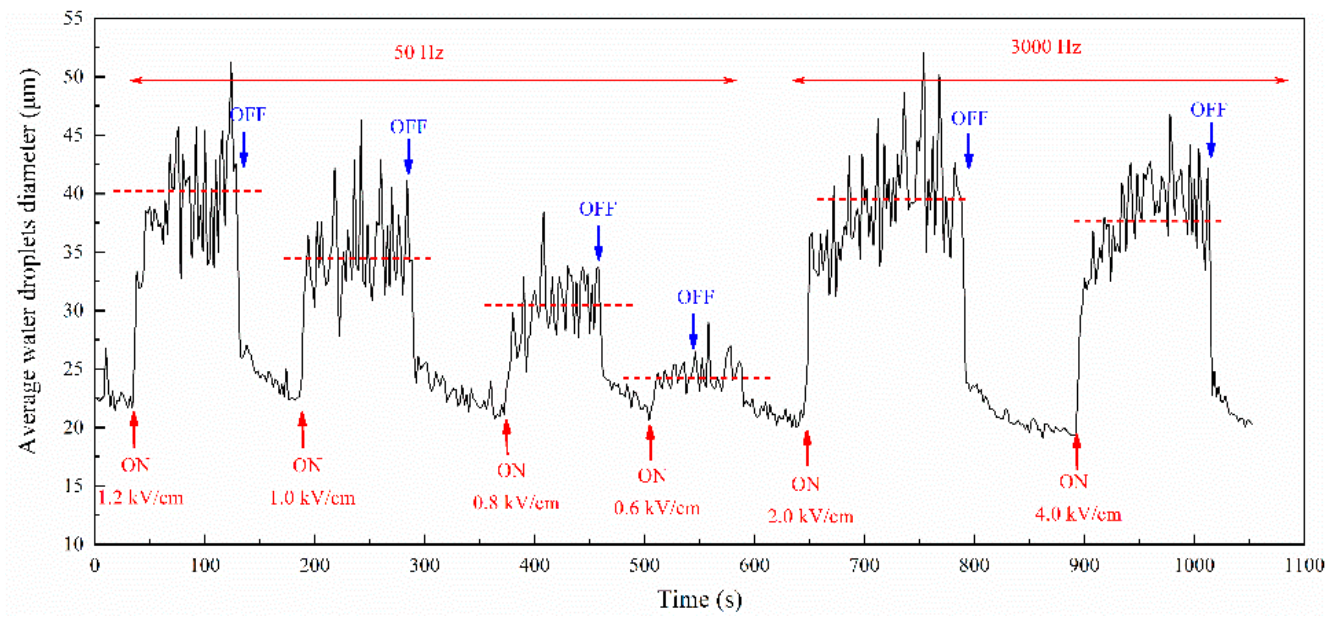

Figure 17. Variations of average water droplet size under different electric field parameters (water content 20\%).

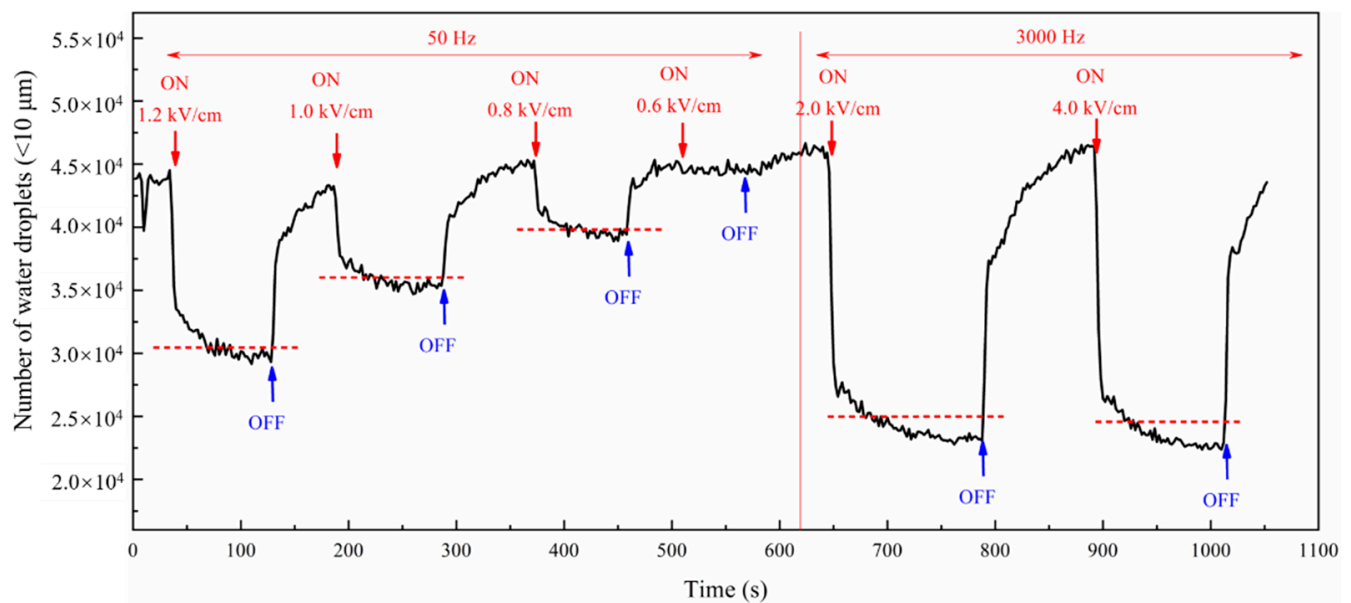

Figure 18. Variations of water droplets number within $10 \mu \mathrm{m}$ (water content 20\%).

(3) Water content: $30 \%$

Figure 19 shows the variations of the average water droplet size in the emulsion with a water content of $30 \%$ and Figure 20 shows the number of water droplets within $10 \mu \mathrm{m}$. As the water content increases, the maximum electric field strength that can be loaded is reduced to $0.8 \mathrm{kV} / \mathrm{cm}$ with an electric frequency of $50 \mathrm{~Hz}$, while the maximum electric field strength can be as high as $2.6 \mathrm{kV} / \mathrm{cm}$ under the high frequency of $3000 \mathrm{~Hz}$. By comparing the demulsification effect among different water contents-10, 20 and 30\%-under a highfrequency electric field $(3000 \mathrm{~Hz})$, the best demulsification performances are almost the same, although the ultimate electric field strengths that can be applied are different.

(4) Water content: $40 \%$

Figure 21 shows the variations of the average water droplet size in the emulsion with a water content of $40 \%$. It is difficult to handle emulsions under a water content of $40 \%$ with metal electrodes. The highest electric field strength that can be achieved is just $0.05 \mathrm{kV} / \mathrm{cm}$ under a $50 \mathrm{~Hz}$ electric frequency. Short circuit occurs once the electric field strength is higher than that value. Although there is no short circuit when the frequency increases 
to $1500 \mathrm{~Hz}$ and $3000 \mathrm{~Hz}$, the maximum electric field strength is only $0.06 \mathrm{kV} / \mathrm{cm}$. There is severe electric field attenuation under high water content. In order to ensure a good demulsification effect under high water content, the insulated metal electrodes should be used with the combination of high-frequency/high-voltage pulsed AC power system.

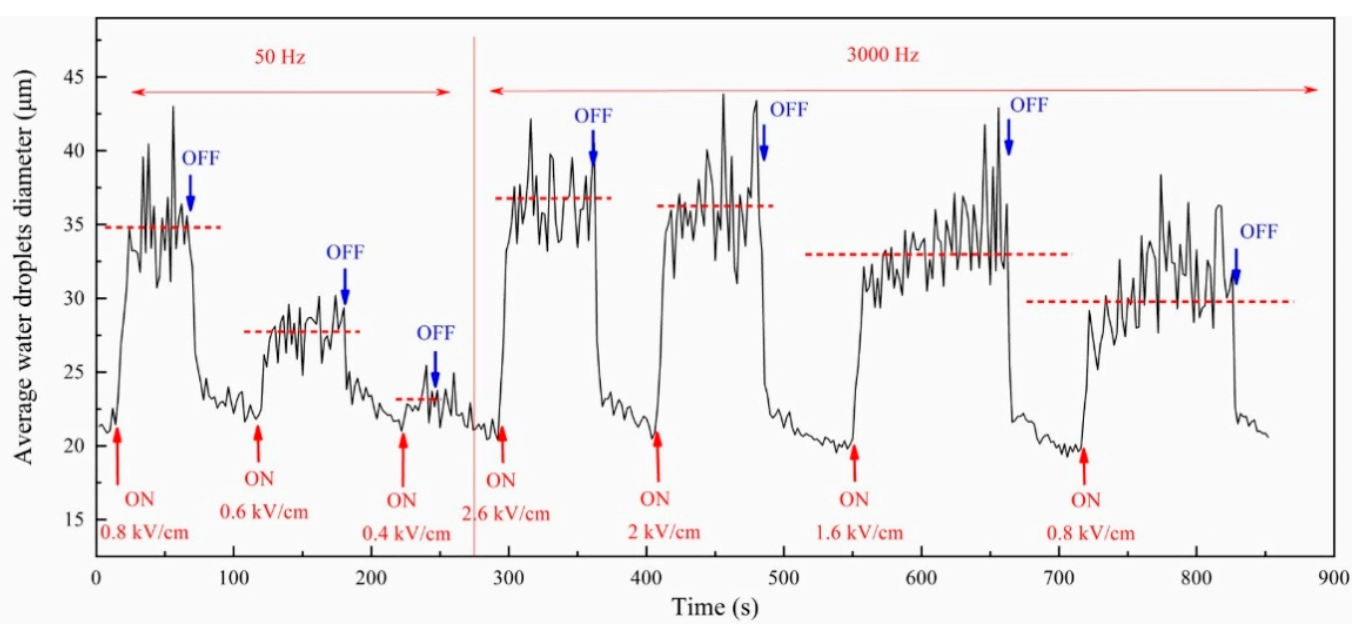

Figure 19. Variations of average water droplet size under different electric field parameters (water content $30 \%$ ).

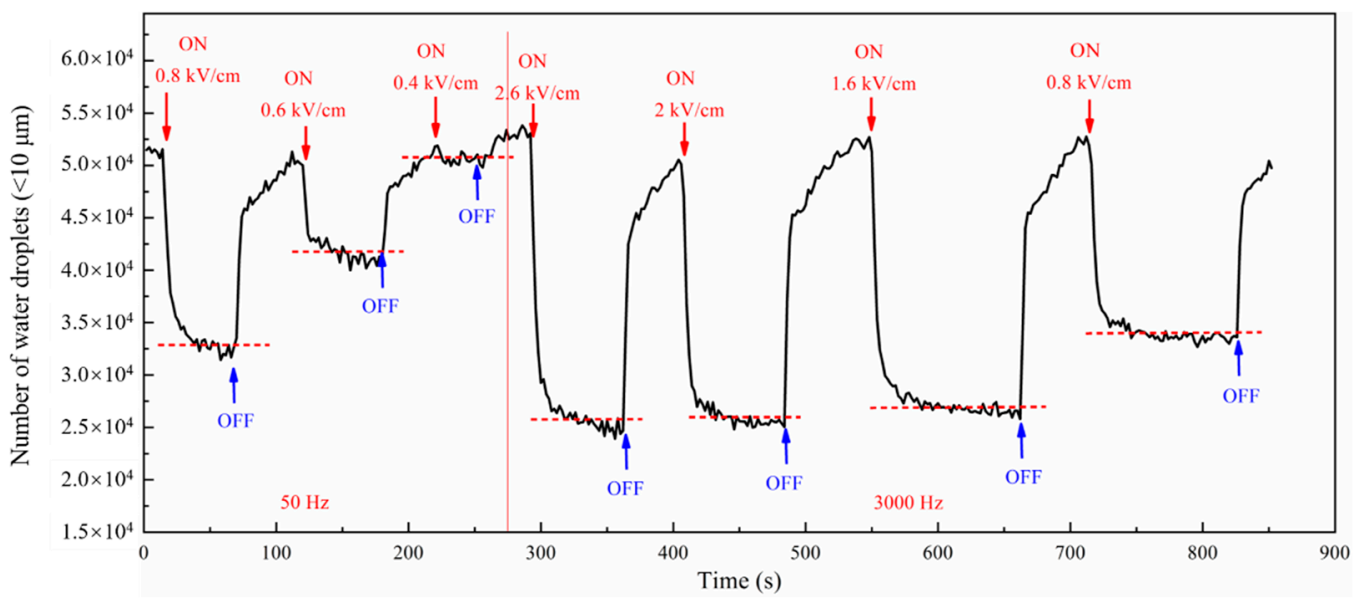

Figure 20. Variations of water droplets number within $10 \mu \mathrm{m}$ (water content $30 \%$ ).

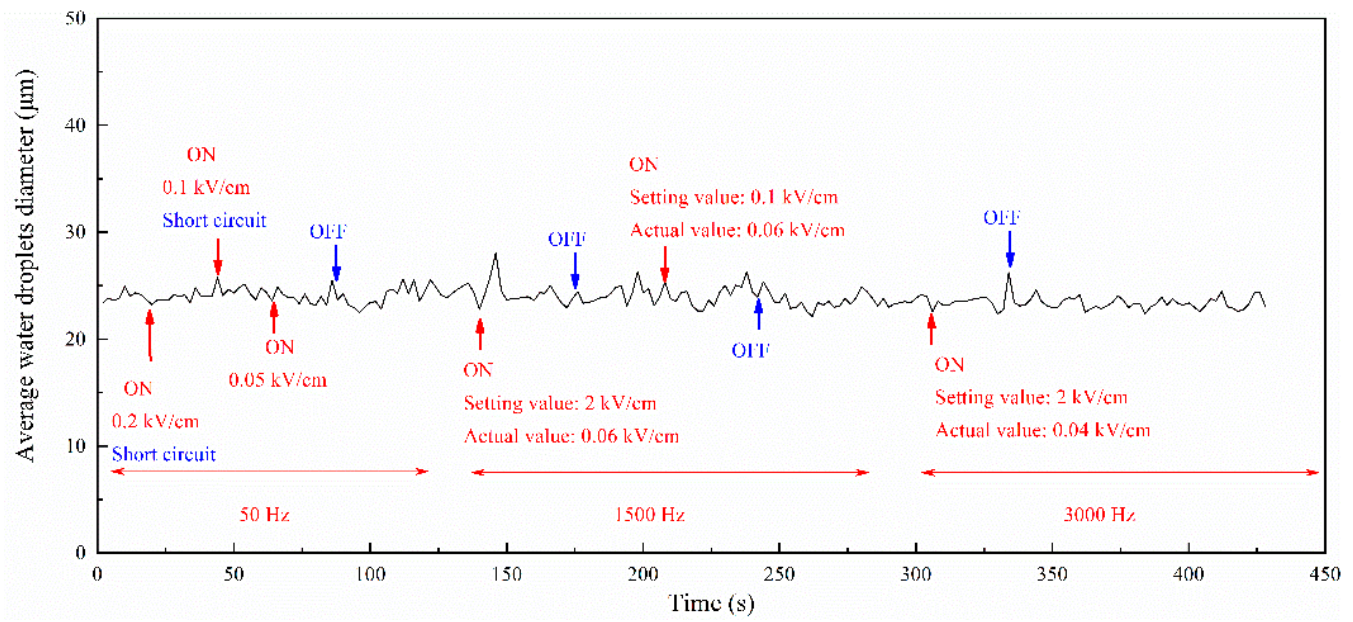

Figure 21. Variations of average water droplet size under different electric field parameters (water content $40 \%$ ). 


\subsection{Effect of the Fluid Velocity}

Based on the above experiment results, the optimized electric field parameters are selected with electric field strength of $3 \mathrm{kV} / \mathrm{cm}$ and frequency of $1500 \mathrm{~Hz}$. The effect of emulsion velocity on the compact electrostatic coalescer demulsification performance is investigated in this part. The emulsion velocities are $0.36,0.53,0.65$ and $0.75 \mathrm{~m} / \mathrm{s}$. More parameters are shown in Table 4.

Table 4. Parameters to study the influence of emulsion velocity.

\begin{tabular}{cccccc}
\hline & \multicolumn{2}{c}{ Flow Conditions } & \multicolumn{2}{c}{ Electric Field Parameters } \\
\hline $\begin{array}{c}\text { Water Content } \\
(\%)\end{array}$ & $\begin{array}{c}\text { Amount of Emulsifier } \\
(\mathbf{g} / \mathbf{L})\end{array}$ & $\begin{array}{c}\text { Rotational Speed of Shear } \\
\text { Dispersing Machine } \\
(\mathbf{r} / \mathbf{m i n})\end{array}$ & $\begin{array}{c}\text { Velocity } \\
(\mathbf{m} / \mathbf{s})\end{array}$ & $\begin{array}{c}\text { Electric Frequency } \\
(\mathbf{H z})\end{array}$ & $\begin{array}{c}\text { Electric Field Strength } \\
(\mathbf{k V} / \mathbf{c m})\end{array}$ \\
& & & 0.36 & & \\
& & 8000 & 0.53 & 1500 \\
& & & 0.65 & \\
\hline
\end{tabular}

Figure 22 shows the variations of the average water droplet diameter with different velocities. The emulsion flow velocity affects the electrostatic coalescence of water droplets mainly by changing the fluid residence time in the electric field and turbulent status. As the velocity increases, the emulsion turbulent state is enhanced. A moderate turbulence state contributes to water droplet coalescence, but it reduces the fluid resident time and thus affects the electrostatic coalescer demulsification efficiency. It shows that decreasing the fluid velocity can obviously promote the coalescence of water droplets by extending the residence time in the electric field. Figure 23 is the chord length distribution of water droplets with different velocities. The trends of the curves between the number of water droplets and the chord length under different velocities are the same. The number of small water droplets is greatly reduced while that of large droplets increases slightly. Additionally, the median number of water droplets grows with the decrease of fluid velocity.

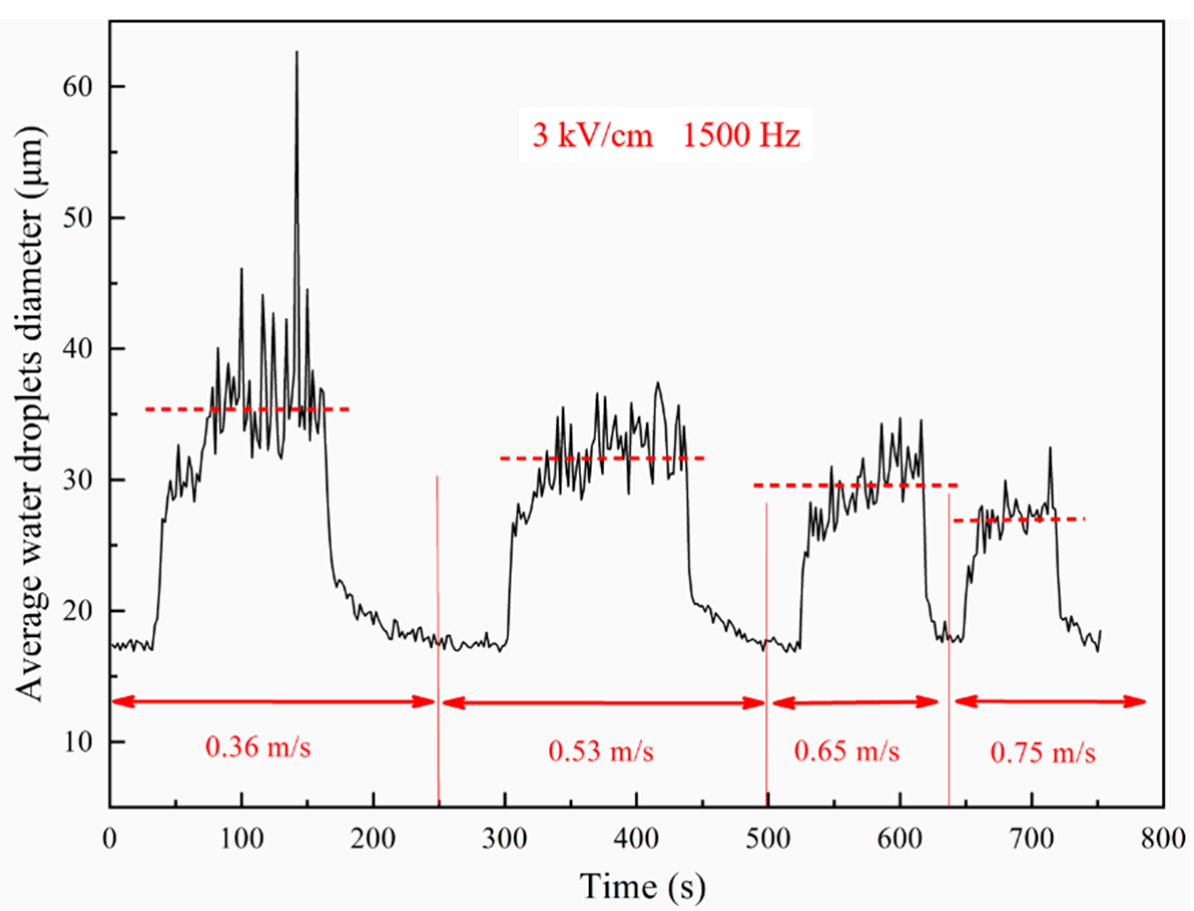

Figure 22. Variations of average water droplet size under different fluid velocities. 


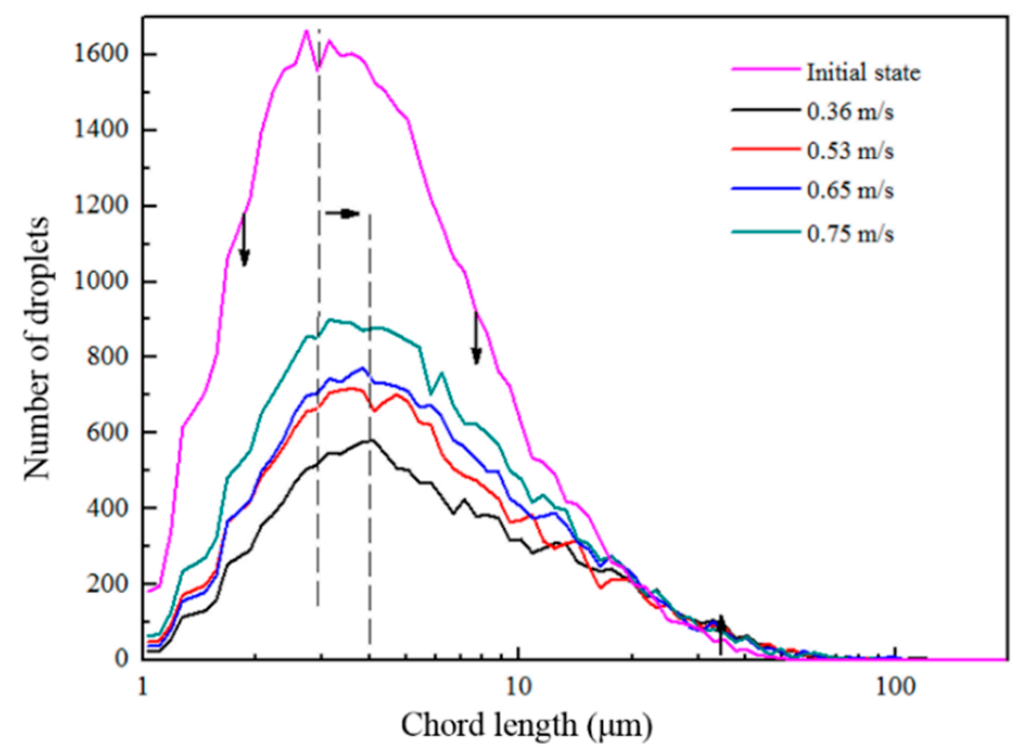

Figure 23. Variations of water droplet number under different chord lengths.

\subsection{Comparison of Performance between the Helical Electrode and Parallel-Plate Electrode}

The comparison of the demulsification performance between the compact electrostatic coalescer with a helical electrode and parallel plate electrode are experimentally studied.

Figure 24 shows the variation of the average water droplet size with the electric field action time under different electric field strengths. The electric frequency is $50 \mathrm{~Hz}$ all the time. Although the maximum electric field strengths with the parallel plate electrode $(2.0 \mathrm{kV} / \mathrm{cm})$ is higher than that with the helical electrode $(1.8 \mathrm{kV} / \mathrm{cm}$ from Figure 12$)$, there is almost no change for the average water droplet size under the action of the electric field if the electric field strength is less than $1.6 \mathrm{kV} / \mathrm{cm}$ with the parallel-plate electrode. When the electric field strength is higher than $1.6 \mathrm{kV} / \mathrm{cm}$, the average water droplet size tends to increase when the power is on. As the electric field strength increases to $1.8 \mathrm{kV} / \mathrm{cm}$, the average water droplet size is increased by $58 \%$ compared to the initial value with the helical electrode, while that with parallel plate electrode is increased by $24 \%$. There are obvious differences between these two different electrodes. The comparison results once again confirm that the demulsification performance of compact electrostatic coalescer can be significantly enhanced by using the helical electrode.

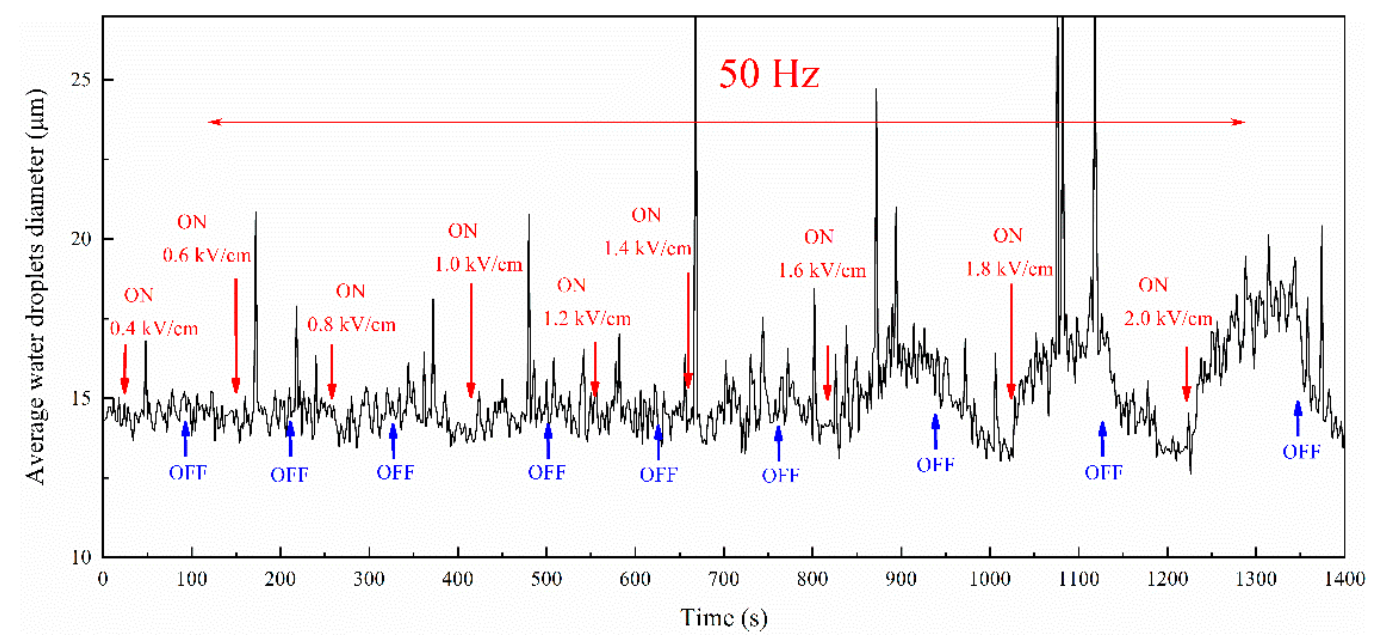

Figure 24. Variations of average water droplet size with parallel plate electrode. 


\section{Conclusions}

The experiment studies were carried out on the demulsification performance of a novel compact electrostatic coalescer with helical electrodes under different flow conditions and electric field parameters. The effects of the electric field strength, frequency, water content and fluid velocity on the demulsification performance were analyzed. The demulsification performance of the novel electrostatic coalescer with a helical electrode was also compared to that with a parallel-plate electrode under the same conditions. The main conclusions are as follows:

(1) The application of helical electrodes can help to increase the average residence time of water droplets in the electric field and the emulsion turbulence is also moderately enhanced. It has a better demulsification performance compared with the electrostatic coalescer with parallel-plate electrodes under the same working conditions. This proves that moderate turbulence is beneficial to promote electrostatic coalescence and the helical electrode can improve the performance of electrostatic coalescence and demulsification.

(2) Under the high-frequency/high-voltage AC-pulsed electric field, the water content that the electrostatic coalescer can treat is also high even if the metal helical electrode is used. It has a better demulsification effect and lower equipment energy consumption under a high-frequency/high-voltage AC-pulsed electric field. The high frequency electric field has more obvious advantages than the power frequency $(50 \mathrm{~Hz})$ electric field. The combination of an electrostatic coalescer with a helical electrode and high-frequency/high-voltage pulse AC power supply technology is feasible

(3) Increasing the residence time of water droplets in the electric field and forming moderate turbulence can both promote electrostatic coalescence. The influence of fluid velocity and the residence time in the electric field should be comprehensively considered when designing an electrostatic coalescer. It is particularly important to determine the appropriate fluid velocity.

Author Contributions: Y.S. wrote the paper and carried out part of the experiment; J.C. determined the structure of this paper and gave suggestions for the improvement of the paper; Z.P. carried out part of the experiment. All authors have read and agreed to the published version of the manuscript.

Funding: This research was funded by the National Natural Science Foundation of China Enterprise Innova-tion and Development Joint Fund (No. U20B2030), the Joint Fund of the Beijing Municipal Education Commission and Beijing Municipal Natural Science Foundation (No. KZ202010017026).

Institutional Review Board Statement: Not applicable.

Informed Consent Statement: Not applicable.

Data Availability Statement: Not applicable.

Conflicts of Interest: The authors declare no conflict of interest.

\section{References}

1. Hamoud, A.A.; Al Ghamdi, S.M. Applications of Multiphase Technologies Optimize Upstream Operations in Saudi Aramco. In Proceedings of the SPE Asia Pacific Oil and Gas Conference, Perth, Australia, 20-22 October 2008.

2. Sun, D.Z.; Jong, S.C.; Duan, X.D.; Zhou, D. Demulsification of water-in-oil emulsion by wetting coalescence materials in stirredand packed-columns. Colloids Surf. A 1999, 150, 69-75.

3. Menon, V.B. Encyclopedia of Emulsion Technology; Marcel Dekker Inc.: New York, NY, USA, 1985; Volume 2.

4. Wu, J.; Xu, Y.; Dabros, T.; Hamza, H. Effect of demulsifier properties on destabilization of water-in-oil emulsion. Energy Fuels 2003, 17, 1554-1559. [CrossRef]

5. Mohammed, R.A.; Bailey, A.I.; Luckham, P.F.; Taylor, S.E. Dewatering of crude oil emulsions. 3. Emulsion resolution by chemical means. Colloids Surf. A 1994, 83, 261-271. [CrossRef]

6. Wang, D.; Yang, D.L.; Huang, C.; Yang, D.Z. Stabilization mechanism and chemical demulsification of water-in-oil and oil-in-water emulsions in petroleum industry: A review. Fuel 2021, 286, 119390. [CrossRef]

7. Lissant, K.J. Demulsification: Industrial Application; Surfactant Science Series 13; Marcel Dekkar: New York, NY, USA, 1983. 
8. Mori, Y.; Tanigaki, M.; Maehara, N.; Eguchi, W. Effect of Frequency in a.c. high voltage on coalescence of water-in-oil emulsion stabilized with sorbitan monooleate type nonionic surfactant. J. Chem. Eng. Jpn. 1994, 27, 340-343. [CrossRef]

9. Simone, L.; Hannisdal, A.; Sjöblom, J. An electrorheological study on the behavior of water-in-crude oil emulsions under influence of a DC electric field and different flow conditions. J. Dispers. Sci. Technol. 2008, 29, 106-114.

10. Simone, L.; Hannisdal, A.; Bjørklund, E.; Sjöblom, J. Electrostatic destabilization of water-in-crude oil emulsions: Application to a real case and evaluation of the Aibel VIEC technology. Fuel 2008, 87, 2572-2581.

11. Eow, J.S.; Mojtaba, G.; Sharif, A.O. Electrostatic enhancement of coalescence of water droplets in oil: A review of the current understanding. Chem. Eng. J. 2001, 84, 173-192. [CrossRef]

12. Ferreira, B.M.S.; Ramalho, J.B.V.S.; Lucas, E.F. Demulsification of water-in-crude oil emulsions by microwave radiation: Effect of aging, demulsifier addition, and selective heating. Energy Fuels 2013, 27, 615-621. [CrossRef]

13. Luo, X.M.; Gong, H.Y.; Cao, J.H.; Yin, H.R. Enhanced separation of water-in-oil emulsions using ultrasonic standing waves. Chem. Eng. Sci. 2019, 203, 285-292. [CrossRef]

14. Reza, Z.; Ahmadun, F.; Luqman, C.; Abdullah, S.; Elnashaie, A.P. Demulsification techniques of water-in-oil and oil-in-water emulsions in petroleum industry. Sep. Purif. Technol. 2016, 170, 377-407.

15. Urdahl, O.; Williams, T.J.; Bailey, A.G. Electrostatic destabilization of water-in-oil emulsions under conditions of turbulent-flow. Chem. Eng. Res. Des. 1996, 74, 158-165.

16. Harpur, I.G.; Wayth, N.J.; Bailey, A.G. Destabilisation of water-in-oil emulsions under the influence of an AC electric field: Experimental assessment of performance. J. Electrost. 1997, 40, 135-140. [CrossRef]

17. Gary, W.S.; Floyd, L.P.; Merle, B.I. Apparatus for Augmenting the Coalescence of Water in a Water-in-Oil Emulsion. US Patent 5,565,078, 15 October 1996.

18. Isabelle, P.; Alexandre, R. Process for Separating Phases of Different Densities and Conductivities by Electrocoalescence and Centrifugation. US Patent 5,643,469, 1 July 1997.

19. Berry, J.P.; Stewart, J.M.; Adrian, G.B. Compact Electrostatic Coalescer. US Patent 6,136,174 A1, 24 October 2000.

20. Urdahl, O.; Nordstad, K.; Berry, P. Development of a New Compact Electrostatic Coalescer Concept. In Proceedings of the SPE Annual Technical Conference and Exhibition, New Orleans, LA, USA, 30 September-3 October 2001.

21. Westra, R.; Akdim, M.R. Compact oil-water separation using the inline electrocoalescer and the cyclonic dewaterer. In Proceedings of the International Petroleum Technology Conference, Bangkok, Thailand, 15-17 November 2011.

22. Grave, E.J.; Olson, M.D.; Menchaca, A.E. Performance testing of an inline electrocoalescer device with medium and heavy crudes. Oil Gas Facil. 2015, 4, 56-65. [CrossRef]

23. Taylor, S.E. Theory and practice of electrically enhanced phase separation of water-in-oil emulsions. Chem. Eng. Res. Des. 1996, 74, 526-540.

24. Christine, N.; Chen, J.Q.; Christine, D. Electrostatic Demulsification on Crude Oil: A State-of-the-Art Review. In Proceedings of the SPE International Oil \& Gas Conference and Exhibition, Beijing, China, 5-7 December 2006.

25. Atten, P. Electrocoalescence of water droplets in an insulating liquid. J. Electrost. 1993, 30, 259-269. [CrossRef]

26. Urdahl, O.; Wayth, N.J.; Fordedal, H. Encyclopedic Handbook of Emulsion Technology; Sjöblom, J., Ed.; CRC Press: Boca Raton, FL, USA, 2001; pp. 679-693.

27. Melheim, J.A.; Matteo, C. Simulation of turbulent electrocoalescence. Chem. Eng. Sci. 2006, 61, 4540-4549. [CrossRef]

28. Li, Q.; Chen, J.Q.; Liang, M. Investigation of water separation from water-in-oil emulsion using high-frequency pulsed AC electric field by new equipment. J. Dispers. Sci. Technol. 2015, 36, 918-923. [CrossRef]

29. Nath, B.; Borthakur, M.P.; Biswas, G. Electric field induced dynamics of viscoplastic droplets in shear flow. Phys. Fluids 2020, 32, 092110. [CrossRef]

30. Ismail, A.S.M.; Menchaca, A.E.; Balk, W.; Akdim, M.R.; Less, S.; Oshinowo, L. High-performance electrostatic coalescer-a novel technology for improving the economics of oil-water separation. In Proceedings of the Abu Dhabi International Petroleum Exhibition and Conference, Abu Dhabi, United Arab Emirates, 9-12 November 2020.

31. Sameer, M.; Rochish, T. Electrocoalescence in non-uniform electric fields: An experimental study. Chem. Eng. Process. 2015, 96, 28-38.

32. Luo, S.R.; Jarrod, S.; Luo, T.F. Effect of electric field non-uniformity on droplets coalescence. Phys. Chem. Chem. Phys. 2016, 18, 29786-29796. [CrossRef] [PubMed]

33. Li, B.; Vivacqua, V.; Wang, J.F. Electrocoalescence of water droplets in sunflower oil using a novel electrode geometry. Chem. Eng. Res. Des. 2019, 152, 226-241. [CrossRef]

34. Zhou, Y.T.; Li, B.; Zhang, M.Y.; Sun, Z.Q.; Wang, Z.B.; Wang, J.J. Effect of dielectrophoresis on the coalescence of binary droplets under a non-uniform electric field. Chem. Eng. Sci. 2020, 224, 115739. [CrossRef]

35. Gong, H.F.; Li, W.L.; Zhang, X.M.; Peng, Y.; Yu, B.; Mou, Y. Effects of droplet dynamic characteristics on the separation performance of a demulsification and dewatering device coupling electric and centrifugal fields. Sep. Purif. Technol. 2021, 257, 117905. [CrossRef] 